\title{
Philosophiques
}

\section{Concurrence fiscale, justice transitionnelle et devoirs de compensation}

\section{Patrick Turmel}

Volume 43, numéro 1, printemps 2016

URI : https://id.erudit.org/iderudit/1036473ar

DOI : https://doi.org/10.7202/1036473ar

Aller au sommaire du numéro

Éditeur(s)

Société de philosophie du Québec

ISSN

0316-2923 (imprimé)

1492-1391 (numérique)

Découvrir la revue

Citer ce document

Turmel, P. (2016). Concurrence fiscale, justice transitionnelle et devoirs de compensation. Philosophiques, 43(1), 133-137.

https://doi.org/10.7202/1036473ar d'utilisation que vous pouvez consulter en ligne.

https://apropos.erudit.org/fr/usagers/politique-dutilisation/ 


\title{
Concurrence fiscale, justice transitionnelle et devoirs de compensation
}

\author{
PATRICK TURMEL
}

Université Laval

Avec Catching Capital. The Ethics of Tax Competition, Peter Dietsch nous offre l'ouvrage le plus complet à ce jour pour réfléchir aux enjeux de justice soulevés par la concurrence fiscale internationale. Non seulement il présente un portrait global et parfaitement informé du problème de l'évitement fiscal, dont on sait qu'il mine aujourd'hui les fondements mêmes de la démocratie et de la justice sociale, mais il nous propose également un cadre normatif et institutionnel pour y faire face et le combattre.

Si la visée ultime est la création d'une organisation fiscale internationale, Dietsch ne se fait pas d'illusion quant à la possibilité de la voir naître à court terme ou sans résistance. C'est la raison pour laquelle il consacre le cinquième et dernier chapitre à la question passionnante de la justice transitionnelle, et donc aux étapes qui pourraient éventuellement mener à la réalisation de la réforme institutionnelle souhaitée.

L'élément le plus important de cette transition serait l'établissement de compensations financières allant des gagnants "nets » aux perdants «nets » de la compétition fiscale. C'est cette idée de devoir de compensation, et ses modalités, que j'aimerais questionner dans cette courte intervention. Mais je dirai d'abord quelques mots du projet plus général de l'ouvrage.

\section{Concurrence fiscale, perspective institutionnelle et agnosticisme normatif}

La concurrence fiscale profite aujourd'hui de l'asymétrie entre une économie mondialisée et un cadre politique encore largement stato-centré. Dans le contexte de cette asymétrie, il y a un fort incitatif pour les individus et les entreprises (qui ont la possibilité, légale ou non, de le faire) de transférer leur capital vers des régimes fiscaux plus cléments. En retour, évidemment, l'incitatif auquel fait face un État particulier est de répondre à cette demande en diminuant ses taux d'imposition, mais également en assouplissant sa réglementation.

Pour Dietsch, cela a deux effets notoires: un effet nocif sur la démocratie - ou sur la possibilité pour une société donnée de se donner de façon souveraine ses propres règles, y compris le choix d'un taux d'imposition élevé sur le capital - et un effet direct sur la justice sociale et l'ampleur des inégalités, non seulement à l'échelle globale, mais également à l'échelle sociale ou nationale.

Pour traiter de ce problème, Dietsch opte pour ce que l'on peut appeler une perspective institutionnelle en philosophie politique, où il accepte que 
certaines caractéristiques, par ailleurs contingentes, du monde social ou institutionnel ne peuvent être ignorées par la réflexion normative. Comme il l'explique, la réflexion normative qu'il mène dans cet ouvrage intègre "un certain nombre de caractéristiques du monde actuel comme paramètres plutôt que comme variables de la réflexion normative» (32). Il refuse donc de situer la réflexion à un niveau d'abstraction tel qu'elle puisse ignorer les institutions et les pratiques qui composent le monde social.

En même temps, Dietsch réitère à plusieurs reprises dans l'ouvrage que son travail ne dépend pas d'une théorie de la justice particulière, ni à l'échelle domestique ni à l'échelle globale. Sans vouloir entrer dans le détail de cette problématique, nous pourrions tout de même nous demander s'il n'y a pas contradiction entre ce que l'on pourrait ainsi appeler son agnosticisme normatif et la perspective institutionnelle qu'il adopte, laquelle n'est d'ailleurs pas sans parti pris théorique marqué. Au minimum, cette perspective suppose une influence de "paramètres " factuels ou empiriques, ce qui semble écarter, contra G. A. Cohen par exemple, toute théorie de la justice dont les principes seraient «insensibles » aux faits. Plus sérieusement, cela écarte la possibilité d'une théorie de la justice qui remette profondément le contexte institutionnel auquel elle s'applique, contra plusieurs théories de la justice globale qui refusent de prendre comme point de départ la structure internationale à l'origine du problème de la concurrence fiscale — ce qu'il reconnaît par ailleurs.

\section{Principes de justice et organisation fiscale internationale}

Dietsch propose une réforme institutionnelle importante, fondée sur deux principes de justice fiscale, s'appliquant non pas directement aux actions d'agents économiques individuels - comme à une entreprise par exemple - mais à la structure des institutions fiscales qui gouvernent les interactions de ces agents et déterminent donc les limites de ce que peuvent faire les États dans le contexte de la concurrence fiscale internationale. Sans revenir sur le détail de ces deux principes, je rappelle tout de même que le principe d'appartenance (membership principle) exige des membres d'un État - citoyens et entreprises - qu'ils assument le fardeau fiscal associé à ce statut et ses bénéfices collectifs (ce qui interdit le resquillage fiscal et exige une véritable transparence fiscale) alors que le principe de contrainte sur les politiques fiscales (fiscal policy constraint) interdit toute politique fiscale qui «est motivée stratégiquement et qui a un impact négatif sur l'autodétermination fiscale agrégée d'autres États» (p. 80).

Pour réaliser ces deux principes, Dietsch propose la création d'une organisation fiscale internationale (International Tax Organisation), qui devrait servir de «forum pour négocier et définir les règles » de la fiscalité internationale, en accord avec les deux principes énoncés (p. I04). Cela signifie notamment, dans le cas de l'implémentation du premier principe, qu'il faille «abolir toutes les règles qui rendent impossibles pour les autres 
pays d'exécuter le principe d'appartenance» (p. I05). Il serait donc interdit dans ce contexte de refuser de partager de l'information pertinente avec les administrations fiscales concernées.

Il s'agira également, dans l'optique d'implémentation du second principe, de s'assurer que les politiques fiscales des États n'ont pas de visée stratégique. L'organisation internationale devra donc servir de forum où les intentions des acteurs pourront être mises en lumière et évaluées. Enfin, ce forum aurait aussi comme tâche de déterminer les devoirs fiscaux des firmes multinationales: quelle administration devrait imposer fiscalement quelle part des profits de ces entreprises? Cette dernière tâche met d'ailleurs en lumière un problème sérieux pour le projet de justice transitionnelle décrit dans le dernier chapitre.

\section{Justice transitionnelle et devoirs de compensation}

Il est intéressant de noter que, s'il discute brièvement des détails de cette organisation fiscale, Dietsch s'attarde davantage aux questions de «justice transitionnelle»- auxquelles il consacre la plus grande part du cinquième et dernier chapitre - qu'à l'implémentation pleine et entière de ses principes de justice. Il passe donc un bon moment à réfléchir à ce que serait une juste transition entre la situation actuelle et l'établissement réussi de l'organisation fiscale internationale annoncée.

L'implication la plus importante pour lui est celle de l'imposition des devoirs de compensation, allant des gagnants «nets» de la concurrence fiscale vers les "perdants nets". Car le véritable problème de la concurrence fiscale a peu à voir avec une opposition stricte entre, d'un côté, des États qui profiteraient de stratégies d'attrait du capital et, de l'autre, des États qui seraient de simples victimes de cette dynamique. D'une certaine façon, la plupart des États sont le paradis fiscal d'un autre: certains bénéficient de la concurrence fiscale dans certains domaines, et en paient le coût dans d'autres. Ajoutons à cela que les véritables perdants sont rarement des populations entières, mais plus souvent la part - importante - de ces populations qui ne profitent pas du système fiscal avantageant ceux qui peuvent se payer les services offerts, et ceux qui évoluent dans cette industrie de l'optimisation ou de l'évitement fiscal. Un écart inéquitable demeure tout de même entre les gagnants nets et les perdants nets de la concurrence fiscale, que devrait mettre en lumière et préciser le calcul des devoirs de compensation allant des premiers aux seconds.

Le premier avantage d'une telle politique transitionnelle serait d'éviter le problème d'action collective qui menace l'institutionnalisation de ses principes de justice. Car, en effet, comment convaincre un État de respecter ces principes, alors qu'il serait avantageux de les ignorer, et cela peu importe ce que font les autres? Et le problème d'action collective est très sérieux de ce point de vue puisque l'implémentation des principes dépend de la mise sur pied d'une organisation internationale dont on ne peut espérer qu'elle voie le jour que si la majorité des États y adhèrent. 
Or, nous dit Dietsch, l'établissement d'un devoir de compensation ne ferait pas face au même type de problème. Il dépendrait uniquement de la volonté politique de chaque État de payer - ou non - cette compensation. Cela dit, ce devoir n'est pas de la même ampleur, et contrairement à ce qu'affirme Dietsch, la raison première pour laquelle même des États «bien intentionnés » risquent fort de ne pas le respecter vient également d'un problème d'action collective: ne serait-il pas injuste de payer notre part si les autres ne font pas de même? Mais Dietsch contourne cette difficulté en présentant cette étape transitionnelle comme une stratégie d'abord rhétorique: "Aussi étrange que cela puisse paraître, nous dit-il, la raison principale de plaider en faveur de ces devoirs de compensation n'est pas réellement de les voir payés» (p. I92).

Pour soutenir cette idée, Dietsch s'inspire du travail de Pablo Gilabert sur la question de la faisabilité ou de l'application en philosophie politique en reprenant la notion de «devoirs dynamiques»:

Si une contrainte de faisabilité nous empêche de faire respecter le devoir $\mathrm{X}$ au temps tI, nous pourrions tout de même avoir un devoir Y de faire quelque chose qui augmentera la probabilité qu'on soit capable de faire respecter le devoir $\mathrm{X}$ au temps t2. Appliqué au contexte présent: si les réformes institutionnelles énoncées dans la première partie ne s'avèrent pas applicables aujourd'hui pour des raisons politiques, il reste un certain nombre de choses que nous pouvons et devrions faire pour rendre leur implémentation possible dans le futur (I93) [ma traduction].

C'est la même intuition qui guide le travail de Dietsch. Pour lui, si le contexte n'est pas mûr pour l'institutionnalisation d'une organisation fiscale internationale, cela ne veut pas dire que rien ne peut être fait pour rendre les conditions de son avènement plus propices. Le calcul des devoirs de compensation joue exactement ce rôle. Cela n'implique pas simplement un devoir de faire advenir les réformes institutionnelles. Si c'est ce que nous devrions retenir de l'idée de Gilabert, nous serions alors clairement aux prises avec un sérieux problème de régression. Car en quoi serait-il plus facile de faire respecter ce devoir? Pour Dietsch, le devoir de compensation permet plutôt d'offrir à différents acteurs un cadre informé (qui perd? qui gagne?) à partir duquel formuler leur revendication ou négocier. Ce qui devrait avoir selon lui une influence positive sur le choix de mettre en branle les réformes institutionnelles souhaitées.

Il y a toutefois, en amont, un problème plus sérieux qui concerne la possibilité même d'établir le calcul des devoirs de compensation et qui met en lumière une tension sérieuse, dans l'ouvrage, entre cette proposition et ce qui a été dit plus tôt à propos des réformes institutionnelles souhaitées. Dans ce dernier chapitre, Dietsch ne fait pas simplement qu'affirmer la nécessité de calculer les droits de compensation, mais offre également certaines pistes pour réaliser ce calcul, affirmant qu'une telle tâche est effectivement réalisable puisqu'il ne s'agit, au fond, que d'un «exercice technique» (p. I95). 
Cela ne contredit-il pas la discussion de la première partie de l'ouvrage, où Dietsch suggère que la présence d'une organisation fiscale internationale est doublement nécessaire à la réalisation du calcul des devoirs de compensation? D'abord, parce qu'il n'y a pas de calcul possible sans transparence fiscale. Ensuite, et ce deuxième point est crucial, parce que le calcul des devoirs de compensation n'est justement pas qu'un exercice technique. Ce calcul dépend de choix ou de conclusions politiques délibérées à propos de la question de savoir où une multinationale devrait payer ses impôts. Il n'y a pas de réponse naturelle ou toute faite à cette question. Ce n'est justement pas simplement une question empirique ou technique. C'est une question éminemment normative qui se pose. Comme il le dit lui-même au deuxième chapitre, la question de savoir quelle part des profits d'une multinationale doit être imposée, et par quelle juridiction, est une "question très épineuse de la pratique fiscale internationale; [...] les détails de la formule en question soulève des enjeux d'équité et de justice distributives entre les pays» (p. I06-I07).

En proposant un calcul «transitionnel» des devoirs de compensation, on court donc le risque non seulement d'imposer un calcul qui fait fi de ces questions normatives épineuses, mais surtout de tracer une voie au risque également de miner la réussite d'une réforme complète des institutions fiscales, en imposant des décisions relatives aux droits et aux devoirs de chacun en amont de la création d'un véritable forum démocratique où ces questions pourraient être soulevées et débattues. Est-ce que ça ne serait pas également déterminer, unilatéralement et avant toute négociation, quels États sont «en dette ", et les décourager à prendre part à la réforme institutionnelle ?

Au final, l'enjeu de l'évasion fiscale, et plus généralement celui de la concurrence fiscale qui en est la condition de possibilité, est à la source du plus sérieux problème de justice de notre temps, mais qui n'est pas traité à la hauteur de son importance par les philosophes politiques. Avec Catching Capital, Peter Dietsch contribue non seulement à corriger cette lacune, mais il propose la référence incontournable à partir de laquelle les philosophes politiques devront désormais réfléchir à ce problème. Nous ne pouvons qu'espérer que son propos soit entendu et discuté le plus largement possible, et notamment hors des cercles universitaires. 